\title{
cryptochrome genes form an oscillatory loop independent of the per/tim loop in the circadian clockwork of the cricket Gryllus bimaculatus
}

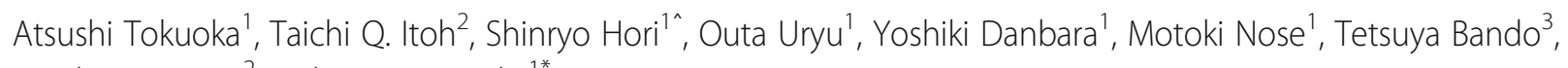
Teiichi Tanimura ${ }^{2}$ and Kenji Tomioka ${ }^{1 *}$ (i)

\begin{abstract}
Background: Animals exhibit circadian rhythms with a period of approximately $24 \mathrm{~h}$ in various physiological functions, including locomotor activity. This rhythm is controlled by an endogenous oscillatory mechanism, or circadian clock, which consists of cyclically expressed clock genes and their product proteins. cryptochrome (cry) genes are thought to be involved in the clock mechanism, and their functions have been examined extensively in holometabolous insects, but in hemimetabolous insects their role is less well understood.

Results: In the present study, the role of cry genes was investigated using RNAi technology in a hemimetabolous insect, the cricket Gryllus bimaculatus. Using a molecular cloning approach, we obtained cDNAs for two cry genes: Drosophila-type cry1 (Gb'cry1) and mammalian-type cry2 (Gb'cry2). Gb'cry2 has six splicing variants, most of which showed rhythmic mRNA expression. Gb'cry ${ }^{\text {RNAi }}$ treatment had only a limited effect at the behavioral and molecular levels, while Gb'cry ${ }^{\text {RNAi }}$ had a significant effect on behavioral rhythms and molecular oscillatory machinery, alone or in combination with Gb'cry ${ }^{\text {RNAi }}$. In Gb'cry / Gb'cry2 double-RNAi crickets, most clock genes showed arrhythmic expression, except for timeless, which retained clear rhythmic expression. Molecular analysis revealed that some combination of Gb'cry1 and Gb'cry2 variants suppressed CLK/CYC transcriptional activity in cultured cells.
\end{abstract}

Conclusion: Based on these results, we propose a new model of the cricket's circadian clock, including a molecular oscillatory loop for Gb'cry2, which can operate independent of the Gb'per/Gb'tim loop.

Keywords: Circadian clock, Clock gene, Cryptochrome, Insect, Molecular oscillatory mechanism

\section{Background}

Circadian rhythms are a biological rhythm with a period of approximately $24 \mathrm{~h}$, which can be observed in various physiological functions of animals, including general activity, hormonal secretion, and metabolism. Rhythmicity is generated by an endogenous mechanism, the so-called circadian clock, which consists of cyclic expression of clock genes and their product proteins. In insects, the core clock components are period (per), timeless (tim),

\footnotetext{
* Correspondence: tomioka@cc.okayama-u.ac.jp

Shinryo Hori Deceased

Deceased

${ }^{1}$ Graduate School of Natural Science and Technology, Okayama University,

3-1-1 Tsushima-naka, Kita-ku, Okayama 700-8530, Japan

Full list of author information is available at the end of the article
}

Clock (Clk), and cycle (cyc) [1, 2]. Clk and cyc encode the transcription factors CLOCK (CLK) and CYCLE (CYC), which form a heterodimer and activate transcription of per and tim. Transcripts of per and tim are subsequently translated to the product proteins PERIOD (PER) and TIMELESS (TIM), which heterodimerize, enter the nucleus, and inhibit transcriptional activity of CLK/CYC. This negative feedback loop produces the $24 \mathrm{~h}$ oscillation. The two cryptochromes, cry1 (or Drosophila-type $c r y$ ) and cry 2 (or mammalian-type cry), are also important components involved in the insect clock. cry proteins are members of the photolyase family, and cry 1 functions as a photoreceptor, resetting the clock through TIM degradation upon exposure to blue light in 
Drosophila and some other insects [3, 4]. cry2 is thought to be a component of the clock, working together with PER to form a feedback machinery [5]. This hypothesis has been supported by a reporter assay using cultured cells $[3,6]$. However, to date the roles of cry genes have been investigated mostly in holometabolous insects, meaning their functions in hemimetabolous insects must be investigated if we are to understand their role in these insects and their functional diversification.

The cricket, Gryllus bimaculatus, is well suited to the study of these questions, since the molecular machinery of the clock has been extensively studied and RNAi can be used to dissect the roles of clock genes $[7,8]$. The clock is located in the optic lobe, and its oscillatory components include Gb'per, Gb'tim, Gb'Clk, and $G b^{\prime} c y c$, as in Drosophila, but their roles differ substantially from those in Drosophila [7]. Similar to the case in Drosophila, Gb'per and Gb'tim are rhythmically expressed and their transcripts increase at night, peaking from early night to midnight $[9,10]$. However, unlike in Drosophila, Gb'cyc, not $\mathrm{Gb}^{\prime} \mathrm{Clk}$, is rhythmically expressed [11, 12]. Doublestranded RNA (dsRNA) treatment of Gb'per and Gb'Clk abolished the locomotor rhythm and the molecular expression rhythms of Gb'per and Gb'tim [9, 11, 13]. However, Gb'tim and Gb'cyc RNAi arrests the rhythmic expression of Gb'per and Gb'tim, but does not prevent behavioral rhythmicity $[10,12]$, suggesting the existence of other oscillatory components. We suggest that cry is a candidate component as, in some insects, CRY functions as a transcriptional repressor by itself, at least in cultured cells [3].

In the present study, we obtained two cry genes in G. bimaculatus by molecular cloning and confirmed that they are homologous to cry1 (Gb'cry1) and cry2 (Gb'cry2) by structural analysis. PCR analysis revealed that there are six splicing variants of Gb'ry2. We then examined their expression profiles and functional roles in the clock machinery by RNAi experiments. RNAi of Gb'ry 2 substantially changed the free-running period of the locomotor rhythm, and the change was further enhanced by $G b^{\prime} c r y 1^{\mathrm{RNAi}}$, suggesting that the $c r y$ genes may be involved in the determination of the free-running period. Although the molecular oscillation of clock genes was mostly halted by Gb'cry1/Gb'cry2 double RNAi, Gb'tim retained its rhythmic expression. Cellular reporter assays revealed that some combination of Gb'ry1 and Gb'ry2 variants represses CLK/CYC transcriptional activity. We propose a unique model for the molecular clock of the cricket that incorporates these new findings and previously described properties of clock genes.

\section{Methods}

\section{Experimental animals}

Third instar nymphs and adult male crickets, Gryllus bimaculatus, were used. These were purchased or obtained from a laboratory colony maintained under standard environmental conditions with a lighting regimen of LD 12:12 (light: 0600-1800; Japan Standard Time) and a constant temperature of $25 \pm 1.0{ }^{\circ} \mathrm{C}$. They were fed laboratory chow and water.

\section{Cloning and structural analysis of the cry genes}

Total RNA was extracted with ISOGEN (Nippon Gene, Tokyo, Japan) from 20 heads of third instar nymphs collected at ZT 10 (ZT stands for zeitgeber time and ZT0 corresponds to lights-on and ZT12 to lights-off). We used $4.5 \mu \mathrm{g}$ of total RNA for reverse transcription to obtain cDNA, using SuperScript II (Invitrogen, Carlsbad, CA, USA). Using the single-stranded cDNA as a template, we performed PCR with degenerate primers deduced from the conserved amino acid sequences among insect Drosophila-type cryptochrome (cry1) and mammaliantype cryptochrome (cry2) homologues. The primers used for cry1 and cry2 were 5'-CAGGAACAAAGAT GGTGGGATAYAAYMGNATG-3' for forward and 5' CCAGTTTCCTGCGCACACNSWCCARTC-3' for reverse, and 5'-TGTTCGTGATCCGAGGACARCCNGC NGA-3' for forward and 5'-CACTGGGATGTACTTT CGGATGWARTCNCCRTT-3' for reverse, respectively. The PCR conditions employed were $30 \mathrm{~s}$ for denaturation at $95{ }^{\circ} \mathrm{C}, 30 \mathrm{~s}$ for annealing at $57{ }^{\circ} \mathrm{C}$, and $1 \mathrm{~min} 30 \mathrm{~s}$ for extension at $72{ }^{\circ} \mathrm{C}$ for 35 cycles with ExTaq DNA polymerase (Takara, Otsu, Japan). The purified fragment was cloned into TOPO-pCR II vector (Invitrogen) and sequenced with BigDye Terminator v3.1 Cycle Sequencing Kit (Applied Biosystems, Foster City, CA, USA). 5' and 3' RACEs for cry 1 and cry 2 were done with GeneRacer kit (Invitrogen) and SMARTer RACE cDNA Amplification kit (Takara) with gene specific primers, 5'-TGGTTCA CAATCCTGCTCAA-3' and 5'-ATGGAGCGCAATCC TATCTG-3', and 5'-CCACTTGGCTAAGGCTTCTG-3' and 5'-GCAGTTCCATCAAGTCAGCA-3', respectively. RACE fragments were purified, cloned and sequenced as mentioned above. Sequences were analyzed by Genetyx ver. 6 (Genetic Information Processing Software, Tokyo, Japan) and BioEdit ver. 7.0.5 (Biological Sequence Alignment Editor, Ibis Therapeutic, Carlsbad, CA, USA). There were six splicing variants in the cricket cry 2 cDNA. For cloning these cDNAs, primer sets of $5^{\prime}$-ATGCAGGTACCATGG GAAGCACACTTGCATT-3' for forward and $5^{\prime}$-ATG CAGCGGCCGCAATTGTGCTGTTGATTTAAAC-3' for reverse, or 5'-ATGCAGGTACCAGTGCTCGTGTGTGT GTTTG-3' for forward and 5'-ATGCAGCGGCCG CAATTGTGCTGTTGATTTAAAC-3' for reverse were used. Exon/intron structure of cry genes were analyzed with genomic DNA sequence data.

Amino acid sequences of CRY1 and CRY2 were aligned using ClustalW in MEGA 7.0. A phylogenetic tree of CRYs was constructed using maximum likelihood 
methods based on JTT matrix-based model in MEGA 7.0. Sequences of known insect cry1 and cry 2 genes were obtained from GenBank.

\section{Measurement of mRNA levels}

Quantitative real-time reverse transcription polymerase chain reaction (qPCR) and reverse transcription polymerase chain reaction (RT-PCR) were used to measure mRNA levels. Total RNA was extracted and purified from six adult male optic lobes with TRIzol Reagent (Invitrogen). To remove contaminating genomic DNA, the total RNA was treated with DNase I. About $500 \mathrm{ng}$ total RNA of each sample was reverse transcribed with random hexamers using PrimeScript RT reagent Kit (Takara). Real-time PCR was performed by Mx3000P Real-Time PCR System (Stratagene, La Jolla, CA, USA) using FastStart Universal SYBR Green Master (Roche, Tokyo, Japan) including SYBR Green with primers designed for Gb'cry1, Gb'cry2, Gb'per (BAG48878), Gb'tim (BAJ16356), and Gb'rpl18a (DC448653) (Table 1). In all cases, a single expected amplicon was confirmed by melting analysis. Quantification was performed based on a standard curve obtained from a known amount of templates. The results were analyzed using the software associated with the instrument.

To estimate expression levels of Gb'ry2 variants, semi-quantitative RT-PCR was performed with Gb'ry2$x 2 a, G b^{\prime} c r y 2-x 4$ and Gb'ry2-x10b primer sets that were designed to detect variants with or without Gb'cry2 exon2a, 4 and 10b, respectively (Table 1). Gb'rpl18a was also used as internal reference. The PCR conditions were $10 \mathrm{~s}$ for denaturation at $98{ }^{\circ} \mathrm{C}, 30 \mathrm{~s}$ for annealing at $52{ }^{\circ} \mathrm{C}$, and $30 \mathrm{~s}$ for extension at $72{ }^{\circ} \mathrm{C}$ for 35 cycles with Emeraldamp ${ }^{\circ}$ MAX PCR Master Mix (Takara, Otsu, Japan) for Gb'ry2 exon2a, $10 \mathrm{~s}$ at $98{ }^{\circ} \mathrm{C}, 30 \mathrm{~s}$ at $55^{\circ} \mathrm{C}$, and $30 \mathrm{~s}$ at $68{ }^{\circ} \mathrm{C}$ for 33 cycles with KOD FX Neo (Toyobo, Osaka, Japan) for exon 4 and $10 \mathrm{~b}, 10 \mathrm{~s}$ at $98{ }^{\circ} \mathrm{C}, 30 \mathrm{~s}$ at $55^{\circ} \mathrm{C}$, and $30 \mathrm{~s}$ at $72{ }^{\circ} \mathrm{C}$ for 28 cycles with Emeraldamp MAX PCR Master Mix for Gb'rpl18a. The PCR samples were electrophoresed on $1.5 \%$ agarose gels in TBE buffer $(89 \mathrm{mM}$ Tris-base pH 7.6, $89 \mathrm{mM}$ boric acid, 2 mM EDTA). Gels were stained with GelRed ${ }^{\mathrm{TM}}$ nucleic acid gel stains (Biotium, CA, USA) and photographed on a $280 \mathrm{~nm}$ UV light box (TP-20MP, ATTO, Tokyo, Japan). The gel images

Table 1 Primers used for qPCR, PCR, dsRNA synthesis, and cloning of coding regions for cell-based assay

\begin{tabular}{|c|c|c|}
\hline Primers & Forward & Reverse \\
\hline \multicolumn{3}{|l|}{ For qPCR } \\
\hline Gb'cry 1 & 5'-AAAGCCAGTTACGCTGATGG-3' & 5'-TGTAATCTCAGGCCATGTCG-3' \\
\hline Gb'cry2 & 5'-AGCACCATCACACACTTCACA-3' & 5'-ACACTCAGCGCAATCCACAC-3' \\
\hline Gb'per & 5'-AAGCAAGCAAGCATCCTCAT-3' & 5'-CTGAGAAAGGAGGCCACAAG-3' \\
\hline Gb'tim & 5'-CCAAAGACAGGAAGCAGACC-3' & 5'-GAATCCCAACACCAAAATGG-3' \\
\hline Gb'rp/18a & 5'-GCTCCGGATTACATCGTTGC-3' & 5'-GCCAAATGCCGAAGTTCTTG-3' \\
\hline \multicolumn{3}{|l|}{ For PCR } \\
\hline Gb'cry2-x2a & 5'-GTGGTATCAACGCAGAAGTA-3' & 5'-TCTAGGCACTGTAGTAGGAA-3' \\
\hline Gb'cry2-x4 & 5'-TTCCGATGCATTTTCATCC-3' & 5'-CGCCAAAGCTTCTGATTCTCC-3' \\
\hline Gb'cry2-x10b & 5'-CTCCTGAGAGTGTGCAACGT-3' & 5'-ACTTGATGGAACTGCTGCCA-3' \\
\hline \multicolumn{3}{|c|}{ For dsRNA synthesis } \\
\hline Gb'cryl\#d1 & 5'-TAATACGACTCACTATAGGGGTITCGACATGGCCTGAGAT-3' & 5'-AATTAACCCTCACTAAAGGGCTTCTGGCGTTGGAAAATGT-3' \\
\hline Gb'cry1\#d2 & 5'-TAATACGACTCACTATAGGGTGGGAACAAGGAAAGACTGG-3' & 5'-AATTAACCCTCACTAAAGGGCGACAATGAGGTGGAGGATT-3' \\
\hline Gb'cry2\#d1 & 5'-TAATACGACTCACTATAGGGCTGCGACAAATAACCCCAAC-3' & 5'-AATTAACCCTCACTAAAGGGCTCTCAGGAGCATTCCAAGG-3' \\
\hline Gb'cry2\#d2 & 5'-TAATACGACTCACTATAGGGAAGCACACTGTGCATTGGTT-3' & 5'-AATTAACCCTCACTAAAGGGCCGTTCTITTCGATGATGCT-3' \\
\hline Gb'per & 5'-TAATACGACTCACTATAGGGCATTCATCGACTTCGTTCACC -3' & 5'-AATTAACCCTCACTAAAGGGCTGAACGCCCAATCATGTCT -3' \\
\hline Gb'tim & 5'-AATTAACCCTCACTAAAGGGGTAAAGAAGATAGAGAGTAT-3' & 5'-AATTAACCCTCACTAAAGGGTTGGAGAGAACTGAAGAGGT-3' \\
\hline$G b^{\prime} C l k$ & 5'-TAATACGACTCACTATAGGGTACATCACGCCATAGCCTTG -3' & 5'-AATTAACCCTCACTAAAGGGGGGATTGCTCTTCTTTGCTG -3' \\
\hline Gb'cyc & 5'-TAATACGACTCACTATAGGGCGTGCACTCGTACACTGAGG-3' & 5'-AATTAACCCTCACTAAAGGGAGGTTCTGCTGCTTCTTTCG-3' \\
\hline DsRed2 & 5'-TAATACGACTCACTATAGGGTCATCACCGAGTTCATGCG-3' & 5'-TAATACGACTCACTATAGGGCTACAGGAACAGGTGGTGGC-3' \\
\hline \multicolumn{3}{|c|}{ For cloning of coding region for cell-based assay } \\
\hline Gb'cryl & 5'-ATGCAGGTACCAAGCCAGTTACGCTGATGGT-3' & 5'-GGGCTCGAGAGTGGTCAAAGCAATTATCAT-3' \\
\hline Gb'cry2\#p1 & 5'-ATGCAGGTACCATGGGAAGCACACTTGCATT-3' & 5'-ATGCAGCGGCCGCAATTGTGCTGTTGATTTAAAC-3' \\
\hline Gb'cry2\#p2 & 5'-ATGCAGGTACCAGTGCTCGTGTGTGTGTTTG-3' & 5'-ATGCAGCGGCCGCAATTGTGCTGTTGATTTAAAC-3' \\
\hline
\end{tabular}

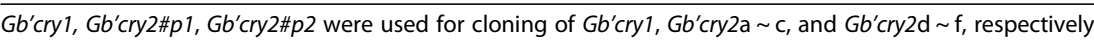


were digital-imaged and quantified by imageJ software (available from https://imagej.nih.gov/ij/). The quantity of the PCR samples were estimated relative to the internal reference gene, Gb'rpl18a, amplified with the same PCR protocol.

For both qPCR and RT-PCR, the values were normalized with those of Gb'rpl18a at each time point. Results of 3-5 independent experiments were used to calculate the mean \pm SEM.

\section{RNAi}

dsRNA for Gb'cry1, Gb'cry2, Gb'per (GenBank/EMBL/ DDBJ Accession No. BAG48878), Gb'tim (BAJ16356), Gb'Clk (AB738083), Gb'cyc (AB762416) and DsRed2 derived from a coral species (Discosoma sp.), were synthesized using MEGAscript High Yield Transcription kit (Ambion, Austin, TX, USA). For Gb'cry1 and Gb'cry2, cDNAs prepared as described above, were used as templates of PCR, which was performed with ExTaq DNA polymerase (Takara). The T7 or T3 containing primers used were listed in Table 1. Amplified Gbcry1\#d1 (575 bp), Gb'cry1\#d2 (515 bp), Gb'cry2\#d1 (463 bp), Gb'cry2\#d2 (423 bp), Gb'per (456 bp), Gb'tim (519 bp), Gb'Clk (407 bp), and Gb'cyc (450 bp) fragments were extracted with phenol/chloroform and precipitated with ethanol then resuspended in Ultra Pure Water (Invitrogen). For DsRed2 dsRNA, linearized DsRed2 fragment was amplified from pDsRed2-N1 (Clontech, Mountain View, CA, USA), with the primers shown in Table 1 . With each of these linearized fragments as a template, RNA was synthesized with T7 or T3 RNA polymerase. Synthesized RNAs were extracted with phenol/chloroform, and suspended in $50 \mu \mathrm{l}$ TE solution after isopropanol precipitation. The yield and quality of RNA were assessed by absorbance using a spectral photometer (Genequant Pro, Amersham Bioscience, Piscataway, NJ, USA) and the same amount of sense and antisense RNA were mixed. The RNAs were denatured for $5 \mathrm{~min}$ at $100{ }^{\circ} \mathrm{C}$ and annealed by a gradual cool down to room temperature. After ethanol precipitation, the obtained dsRNA was suspended in Ultra Pure Water (Invitrogen) and adjusted to the final concentration of $20 \mu \mathrm{M}$. The dsRNA solution was stored at $-80^{\circ} \mathrm{C}$ until use. $760 \mathrm{nl}$ of dsRNA solution was injected with a nanoliter injector (WPI, Sarasota, FL, USA) into the abdomen of adults anesthetized with $\mathrm{CO}_{2}$.

\section{Assays in cultured cells}

The coding sequences of cry genes, Gb'cry1 and Gb'ry2a-f, were subcloned individually into pAc5.1BV5/His (Invitrogen, Carlsbad, CA). Primers used for cloning are listed in Table 1. Cultured Drosophila S2 cells were plated in 24-well tissue culture plates in Shields and Sang M3 insect medium (Sigma, St Louis, MO) supplemented with $12.5 \%$ fetal bovine serum (Biowest,
Canada) and antibiotics (12.5 U/ml penicillin, $12.5 \mathrm{mg} / \mathrm{ml}$ streptomycin; GIBCO, Grand Island, NY). S2 cells were transfected by Effectene Transfection Reagent (Qiagen, Hilden, Germany) with $100 \mathrm{ng}$ of $p A c t-C l k$ as an activator [14], $100 \mathrm{ng}$ of Drosophila tim-luc [14], which contains a typical E-box [15] showing a relatively high expression [16], and $10 \mathrm{ng}$ of pAc5.1-Rluc [17] as a positive control for luciferase activity, along with a combination of $500 \mathrm{ng}$ of $p A c 5.1-G b^{\prime} c r y 1$ and $p A c 5.1-G b^{\prime} c r y 2 \mathrm{a} \sim \mathrm{f}$. As needed, the empty vector $p A c 5.1 B$-V5/His was used instead of the vector constructs of $G b^{\prime} c r y 1$ and Gb'ry2 genes to ensure that an equal amount of DNA was used for transfection in each well. Forty-eight hours after transfection, luciferase assays of transfected cells were carried out using the DualLuciferase $^{\bullet}$ Reporter Assay System (Promega Corporation, Madison, Wisconsin, USA) and then normalized to Rluc activity as a control for transfection efficiency. Assays were performed at least three times.

\section{Behavioral analysis}

Locomotor activities were recorded in the same way as described previously [9]. Briefly, the final instar nymphs or adult crickets were individually housed in a transparent plastic box $(18 \times 9 \times 4.5 \mathrm{~cm})$ with a rocking substratum. The number of substratum rocking was recorded every $6 \mathrm{~min}$ by a computerized system. Food and water were provided ad libitum. The actographs were placed in an incubator (MIR-153, Sanyo Biomedica, Osaka, Japan) in which temperature was kept at $25 \pm 0.5{ }^{\circ} \mathrm{C}$ and lighting conditions were maintained by a cool white fluorescent lamp connected to an electric timer. The light intensity was 600-1000 lux at the animal's level, varying with the proximity to the lamp. The raw data were displayed as conventional double-plotted actograms to judge activity patterns, and the free-running period was calculated by the chi-square periodogram [18] with Actogram J (freely available at http://acto gramj.neurofly.de/) [19]. If a peak in the periodogram appeared above the 0.05 confidence level (alpha $=0.005$ ), the power value (height of the peak above the confidence level) was greater than or equal to 10 , and the width of the peak was greater than or equal to 2, the period for the peak was designated statistically significant [20].

\section{Statistical analysis}

The one-way analysis of variance (ANOVA) followed by a post hoc Tukey-test was used to compare the differences in means of mRNA levels between the different time points and the differences in means of free-running periods and phase angle differences between insect groups with different treatments. To compare the means of two groups, $t$-test was used. Dunnett's-test was also used for multiple comparisons, where applicable. Significance of the daily and circadian rhythmicity in mRNA 
expression was examined by the single cosinor method [21], fitting a cosine curve of $24 \mathrm{~h}$ period using Time Series Analysis Serial Cosinor 6.3 (Expert Soft Technologie, Richelieu, France). In all statistical tests, the significance level was set at $P<0.05$.

\section{Results}

\section{Cloning and structural analysis of Gb'cry1 and $G b^{\prime}$ 'cry 2}

cry 1 and cry 2 homologues were cloned from the optic lobe of G. bimaculatus using a degenerate PCR strategy with degenerate primers based on conserved amino acid sequences from insects cry 1 and cry 2 homologues. A Gryllus bimaculatus cry1 (Gb'cry1) fragment of $1116 \mathrm{bps}$ and a cry2 (Gb'cry2) fragment of $876 \mathrm{bps}$, including the FAD-binding 7 domain and a part of PhrB, was first obtained. By 3' rapid amplification of cDNA ends (RACE) and 5' RACE, we obtained a full length of $1937 \mathrm{bp}$ Gb'ry1 cDNA (GenBank/EMBL/DDBJ Accession No. LC202047). The Gb'cry1 cDNA had 189 bp 5'-untranslated regions (UTR) and 131 bp 3'-UTR. Genomic DNA analysis revealed that G'cry1 consists of nine exons, and no splicing variants were detected by RT-PCR. The putative product protein $G b^{\prime} \mathrm{CRY} 1$ consists of 537 amino acid residues (Fig. 1a).

As to Gb'ry2, we obtained six variants of cDNAs, those are G'cry2a $\sim \mathrm{f}$ (GenBank/EMBL/DDBJ Accession No. LC202048, LC202049, LC202050, LC202051, LC202052, LC202053) (Fig. 1a). The lengths of cDNA varied among the variants. Their structures are illustrated in Fig. 1a with deduced proteins. With genomic sequence analysis, the Gb'rry2 gene was shown to consist of 11 exons and the splicing variants were shown to commonly include exons 1-3 and 5-11 with variable lengths of exons 2, 6 and 10 . Exceptionally, a variant Gb'ry2e lacked exon 4. Gb'rry2a c had a short exon 2 lacking the 2a region, Gb'ry2c with short exon 6 lacking 6a region, and G'bry2a, d with longer exon 10 including a 10b region. From these mRNAs six different proteins are deduced. $G b^{\prime}{ }^{\prime} \mathrm{CRY} 2 \mathrm{a} \sim \mathrm{c}$ lack the photolyase $\alpha / \beta$ domain, because the start codon is located in middle of exon $4, G b^{\prime} C R Y 2 c$ has a shorter N-terminal region lacking exon $6 a$ region, and $G b^{\prime} C R Y 2 a$ and $-d$ have a shorter C-terminal region because of stop codon in exon $10 \mathrm{~b} . G b^{\prime} C R Y 2 e$ is the smallest lacking most part and consists of only 202 amino acid residues, while Gb'CRY2f is the largest, includes all coding regions, and consists of 587 amino acid residues.

A BLAST database search indicated that the amino acid sequence of $G b^{\prime} \mathrm{CRY} 1$ has $55-57 \%$ identities along the entire length of the protein with those of CRY1 characterized in other insects, including the fruit fly Drosophila melanogaster (GenBank/EMBL/DDBJ accession No. AAF55649.1), the monarch butterfly, Danaus plexippus (EHJ63675.1), the silkmoth, Antheraea pernyi (AAK11644.1), and the mosquito, Anopheles gambiae
(ABB29886.1, Table 2). The longest Gb'CRY2f has $62-79 \%$ identity along the entire length of the protein with those of known insect CRY2s and mouse CRYs, including the monarch butterfly, D. plexippus (EHJ74426.1), the silkmoth, A. pernyi (ABO38435.1), the honeybee, Apis mellifera (NP_001077099.1), the mosquito, A. gambiae (ABB29887.1), the red flour beetle, Tribolium castaneum (EFA04537.1) and the mouse, Mus musculus (CRY1, NP_031797.1, CRY2, NP_034093.1) (Table 2).

The deduced proteins G $b^{\prime} \mathrm{CRY} 1$ and Gb'CRY2f commonly had five highly conserved regions that are characteristic of CRY proteins (Fig. 1a): (i) DNA photolyase $\alpha / \beta$ domain, (ii) FAD-binding 7 domain, (iii) RD-2b domain, which is necessary for nuclear localization and the repression of CLOCK: BMAL-mediated transcription, (iv) inhibition of CLOCK-ARNTL-mediated transcription (ICAT) domain, and (v) PhrB domain. Identity of these domains had $51-69 \%$ in $G b^{\prime}$ CRY1, while those in Gb'CRY2f had high identity of $52-100 \%$ and also share high identity with mouse CRY1 and CRY2 (Table 2). A phylogenetic tree based on the amino acid sequences of CRYs from known insects and some vertebrates revealed that $G b^{\prime} \mathrm{CRY} 1$ and Gb'CRY2f form separate clades with CRY1 and CRY2 in other insects (Fig. 1b).

\section{Expression profiles of $G b^{\prime} c r y 1$ and $G b^{\prime} c r y 2$ mRNA in the optic lobe}

To examine whether Gb'ry1 and Gb'cry2 transcript oscillated in the optic lobe, their mRNA levels under LD12:12 were measured using $\mathrm{qPCR}$ in the adult male crickets. Primers used for qPCR were shown in Fig. 1a and Table 1. As shown in Fig. 2a, Gb'cry1 appeared to be constitutively expressed, and no clear daily rhythm was observed (ANOVA, $F_{5,23}=1.03, P>0.42$; cosinor, $P>0.05$ ). For Gb'cry2, rhythmic mRNA expression was found with a peak during the midnight (ZT18, ANOVA, $\mathrm{F}_{5,21}=21.95$, $P<0.01$; cosinor, $P<0.01$ ) when all the splicing variants were amplified with primers designed at exon 11 (Fig. 2b). The amplitude of the rhythm was approximately 4-fold.

We then examined the expression levels of G'cry2a $\sim \mathrm{c}$ and Gb'ry $2 \mathrm{~d} \sim \mathrm{f}$ with short and long exon 2 using a primer set Gbcry2- $x 2 a$ (Table 1), respectively, in the optic lobe at ZT 6 and 18 by a semi-quantitative method, RTPCR. Both of Gb'ry2a $\sim \mathrm{c}$ and Gb'ry2d $\sim \mathrm{f}$ mRNAs with short and long exon 2, respectively, showed a higher level at ZT18, but the expression level was much greater in the latter (Fig. 2c; ANOVA, $F_{3,11}=454.26, P<0.01$ ). When the levels of Gb'cry2a,d and Gb'cry2b, c, e, f with long and short exon 10, respectively, were examined using a primer set Gb'ry2-x10b (Table 1), both were again higher at ZT18 but the levels of Gb'rry2b, c, e, f were higher (Fig. 2c; ANOVA, $\left.F_{3,11}=110.3, P<0.01\right)$. These results suggest that the Gb'ry2f was expressed most abundantly. We also compared the levels of cry2e that lacked exon 4 and 

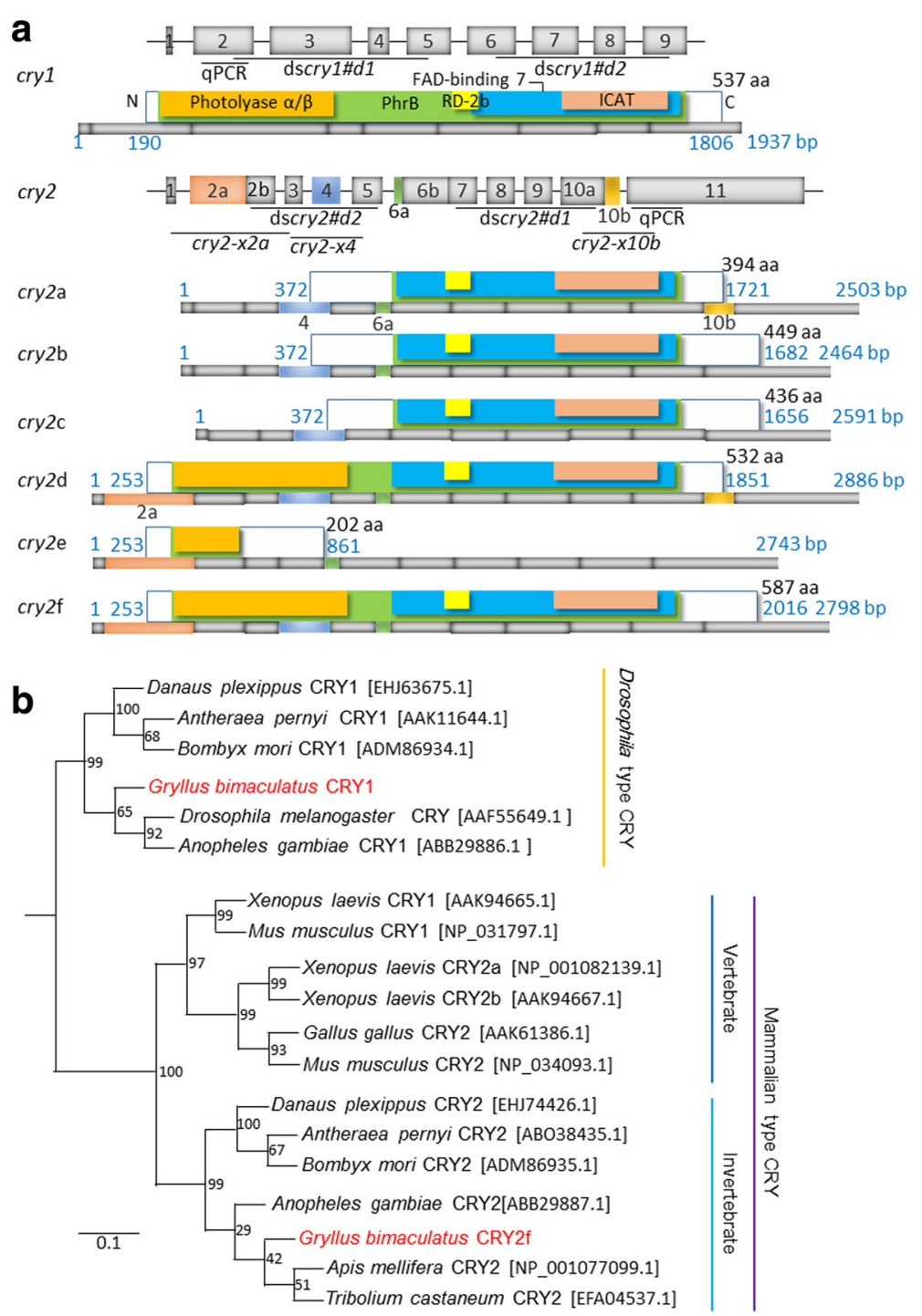

Fig. 1 Sequence alignments and a phylogenetic tree of Gb'cry1 and Gb'cry2. a Schematic structure of Gb'cry 1 and Gb'cry2 cDNAs and their deduced product proteins, showing conserved domains, photolyase a/ß (orange), PhrB (green), RD-2b (yellow), FAD-binding 7 (blue), and inhibition of CLOCK-ARNTL-mediated transcription (ICAT) domain (pink). At the top of schemes of each gene exon/intron structure is illustrated. There are six splicing variants for $G b^{\prime} C R Y 2$, of various lengths. The bars under each protein scheme show CDNA structure and the colored portions indicate the respective exons. Numbers shown at the right of protein illustration indicate the number of amino acid residues (aa) and the blue numbers indicate the number of base pairs. The regions used for qPCR and dsRNA synthesis are also shown. cry $2-x 2 a,-x 4$, and $-x 10 b$ are for PCR for amplification of variants with or without exon 2a, 4, and 10b, respectively. $\mathbf{b}$ A phylogenetic tree of known insect and vertebrate CRY proteins. CRY amino acid sequences were analyzed, and the phylogenetic tree was inferred by the maximum likelihood method in MEGA 7.0. The GenBank or RefSeq accession numbers are indicated in brackets. The reference bar indicates the distance as number of amino acid substitutions per site

Gb'cry2a $\sim$ d,f that included exon 4 using a primer set Gb'ry2-x4 (Table 1). The results showed that they again showed higher expression at ZT18 (ANOVA, $\mathrm{F}_{3,11}=$ 167.36, $P<0.01$ ) and that most of expressed Gb'cry2 variants were those including exon 4 and the cry2e expression was only negligible (Fig. 2c).

\section{RNAi suppresses $G b^{\prime}$ 'cry1 and $G b^{\prime}$ cry2 expression}

We examined the effects of dsRNA of Gb'ryl (dsGb'cry1) and Gb'ry 2 (dsGb'ry2) on respective transcript levels, by measuring their mRNA levels in the optic lobe of the adult male crickets by qPCR. dsGb'ry2\#d1 and dsGb'cry2\#d2 were synthesized for the region included in all splicing variants (Fig. 1a). Treatment of dsGb'cry1\#d1 or dsGb'cry1\#d2 significantly reduced Gb'ry 1 mRNA levels throughout the day (Fig. 2a). In the crickets injected with dsGb'ry $\# d 1$ or dsGb'ry2\#d2, the levels of Gb'ry2 mRNA were knocked down to nearly the basal level of that of intact crickets; although a small but significant fluctuation was observed (ANOVA: $\mathrm{F}_{5,19}=5.44, P<0.05$ 
Table 2 Overall amino acid identity (\%) of whole sequence and functional domains of Gryllus bimaculatus CRY1 and CRY2f with their insect and mammalian orthologues

\begin{tabular}{|c|c|c|c|c|c|c|}
\hline Species & Whole sequence & DNA photolyase & FAD binding 7 & $\mathrm{RD}-2 \mathrm{~b}$ & ICAT & PhrB \\
\hline \multicolumn{7}{|l|}{ Gb'CRY1 } \\
\hline Drosophila melanogaster CRY & 55 & 59 & 59 & 51 & 63 & 57 \\
\hline Danaus plexippus CRY1 & 56 & 56 & 64 & 54 & 69 & 58 \\
\hline Antheraea pernyi CRY1 & 57 & 59 & 63 & 48 & 69 & 59 \\
\hline Anopheles gambiae CRY1 & 56 & 59 & 61 & 51 & 65 & 58 \\
\hline \multicolumn{7}{|l|}{$G b^{\prime} C R Y 2 f$} \\
\hline Danaus plexippus CRY2 & 67 & 82 & 82 & 75 & 81 & 80 \\
\hline Antheraea pernyi CRY2 & 71 & 80 & 85 & 82 & 84 & 81 \\
\hline Apis mellifera CRY2 & 77 & 85 & 92 & 96 & 91 & 87 \\
\hline Anopheles gambiae CRY2 & 73 & 82 & 91 & 100 & 89 & 85 \\
\hline Tribolium castaneum CRY2 & 79 & 79 & 91 & 96 & 89 & 85 \\
\hline Mus musculus CRY1 & 64 & 65 & 82 & 79 & 79 & 74 \\
\hline Mus musculus CRY2 & 62 & 65 & 79 & 75 & 74 & 71 \\
\hline
\end{tabular}
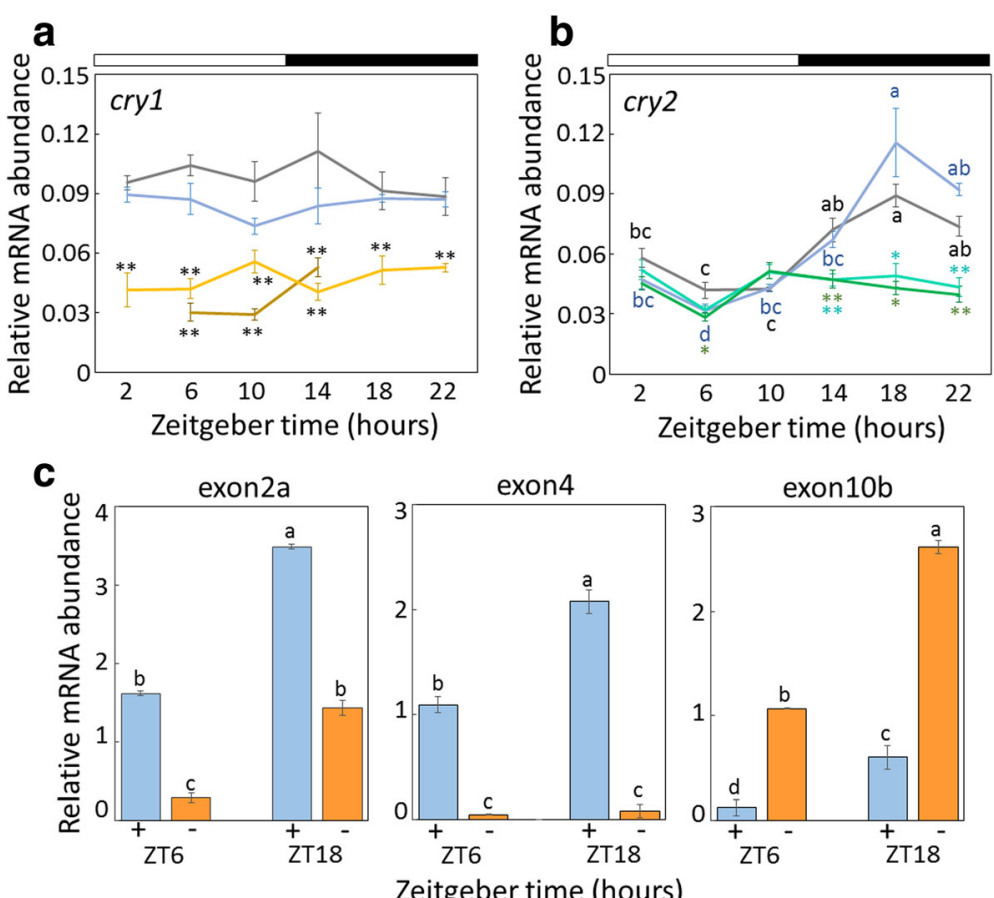

Fig. 2 Expression profiles of Gb'cry1 (a) and Gb'cry2 mRNA (b, c) in Gryllus bimaculatus optic lobes under LD 12:12. In (a) and (b), abundance of Gb'cry1 and Gb'cry2 mRNA is shown for intact (blue), dsDsRed2 (gray), dsGb'cry1\#d1 (yellow), dsGb'cry1\#d2 (dark yellow), dsGb'cry2\#d1 (dark green), dsGb'cry2\#d2 (green) treated crickets. Total RNA was extracted from the optic lobes that were collected at 4-hour intervals starting at $2 \mathrm{~h}$ after lights-on (ZT 2). White and black bars indicate light and dark, respectively. Gb'cry 1 was rather constitutively expressed. Gb'cry 2 showed rhythmic expression in intact and DsRed2 ${ }^{\mathrm{RNAi}}$ crickets (cosinor, $P<0.01$ ), while the rhythm disappeared when treated with dsGb'cry2 (cosinor, $P>0.05$ ). Note that dsRNA significantly reduced mRNA levels of respective genes, while DsRed2 dsRNA had no significant effect. Asterisks indicate significant difference between control (DsRed2 ${ }^{\text {RNAi)})}$ and treated crickets ( ${ }^{*} P<0.05,{ }^{* *} P<0.01$, Dunnett's test). c Semi-quantification of Gb'cry2 mRNA levels at ZT6 and ZT18 with (b/ue) and without (orange) exon 2a, 4, or 10b. Different lower case letters indicate that values are significantly different with each other (ANOVA followed by Tukey-test, $P<0.05$ ). For $(\mathbf{a}-\mathbf{c}$ ), data collected from three or five independent experiments were averaged and plotted as mean $\pm \mathrm{SEM}$. The abundance of Gb'rp/18a mRNA was used as an internal reference 
for dsGb'cry2\#d1; $F_{5,23}=3.18, P<0.05$ for dsGb'cry2\#d2, Fig. 2b), no significant daily rhythm was detected on single cosinor analysis $(P>0.05)$.

\section{Effects of Gb'cry1 and Gb'cry2 RNAi on the locomotor rhythm}

To examine the role of G'cry1 and Gb'cry 2 in circadian locomotor rhythm regulation, locomotor activity was recorded in adult males injected with dsGb'ry1 (dsGb'ry1\#d1, $N=19$; dsGb'cry1\#d2, $N=22$ ) or dsGb'ry2 (dsGb'ry2\#d1, $N=37$; dsGb'ry2\#d2, $N=22$ ). Since similar results were obtained in crickets treated with two different dsRNAs for Gb'ry1 and Gb'ry2, the results were pooled. We used DsRed $2^{\mathrm{RNAi}}$ crickets $(n=21)$ as a negative control. As was the case for DsRed2 ${ }^{\mathrm{RNAi}}$ crickets, all of the $G b^{\prime} c r y 1^{\mathrm{RNAi}}$ and the Gb'ry $2^{\mathrm{RNAi}}$ crickets exhibited a nocturnal activity rhythm under LD12:12, with a major peak at lights-off and a minor peak at lights-on (Fig. 3). In the ensuing constant darkness (DD), the rhythm free-ran, except in $4 G^{\prime} b^{\prime} r y 2^{\mathrm{RNAi}}$ crickets which became arrhythmic, and the free-running periods varied with treatments (Figs. 3 and 4). The Gb'cry $1^{\text {RNAi }}(n=16)$ and

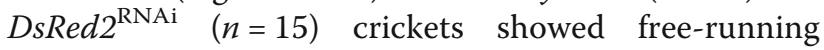
periods shorter than $24 \mathrm{~h}$, averaging $23.58 \pm 0.25 \mathrm{~h}$ and $23.71 \pm 0.24 \mathrm{~h}$, respectively. However, the free-running period varied widely in $G b^{\prime} c r y 2^{\mathrm{RNAi}}$ crickets $(n=33)$ : the average period was $24.12 \pm 0.34 \mathrm{~h}$ ranging from $23.2 \mathrm{~h}$ to $25.0 \mathrm{~h}$. When treated doubly with dsGb'ry 1 and dsGb'ry2, the crickets were all rhythmic but showed a wider range of free-running period (22.4-25.8 h) than Gb'ry ${ }^{\mathrm{RNAi}}$ crickets. Notably, some of the $G b^{\prime} c r y 2^{\mathrm{RNAi}}$ crickets (dsG'cry2\#d1,n $=3$; dsGb'ry2\#d2, $n=2$ ) showed a rhythm that split into two components in the free-running condition (as exemplified in Fig. 3d) and that individuals with the free-running periods longer than $24 \mathrm{~h}$ often became arrhythmic (dsGb'cry2\#d1, $N=4$ ) or only weakly rhythmic (dsGb'cry2\#d1, $n=8$; dsGb'cry2\#d2, $n=10)$ in due course (as exemplified in Fig. 3d, j).

\section{Effects of $G b^{\prime} c r y 1$ and $G b^{\prime} c r y 2$ RNAi on the molecular clockwork}

We then examined the effects of RNAi of G'cryl and Gb'cry2 on Gb'per and Gb'tim genes, using dsGb'cry1\#d1 and dsGb'ry2\#d1. In intact crickets, transcripts of G'per and Gb'tim showed a rhythmic expression to peak around midnight (ZT18). These expression profiles were similar to those reported previously [9-11]. When Gb'cry1 was knocked-down by RNAi, Gb'cry2 and Gb'tim maintained a rhythmic expression (ANOVA, $F_{5,24}=$ 15.10, $P<0.01$; cosinor, $P<0.05$ for G'́ry2 and

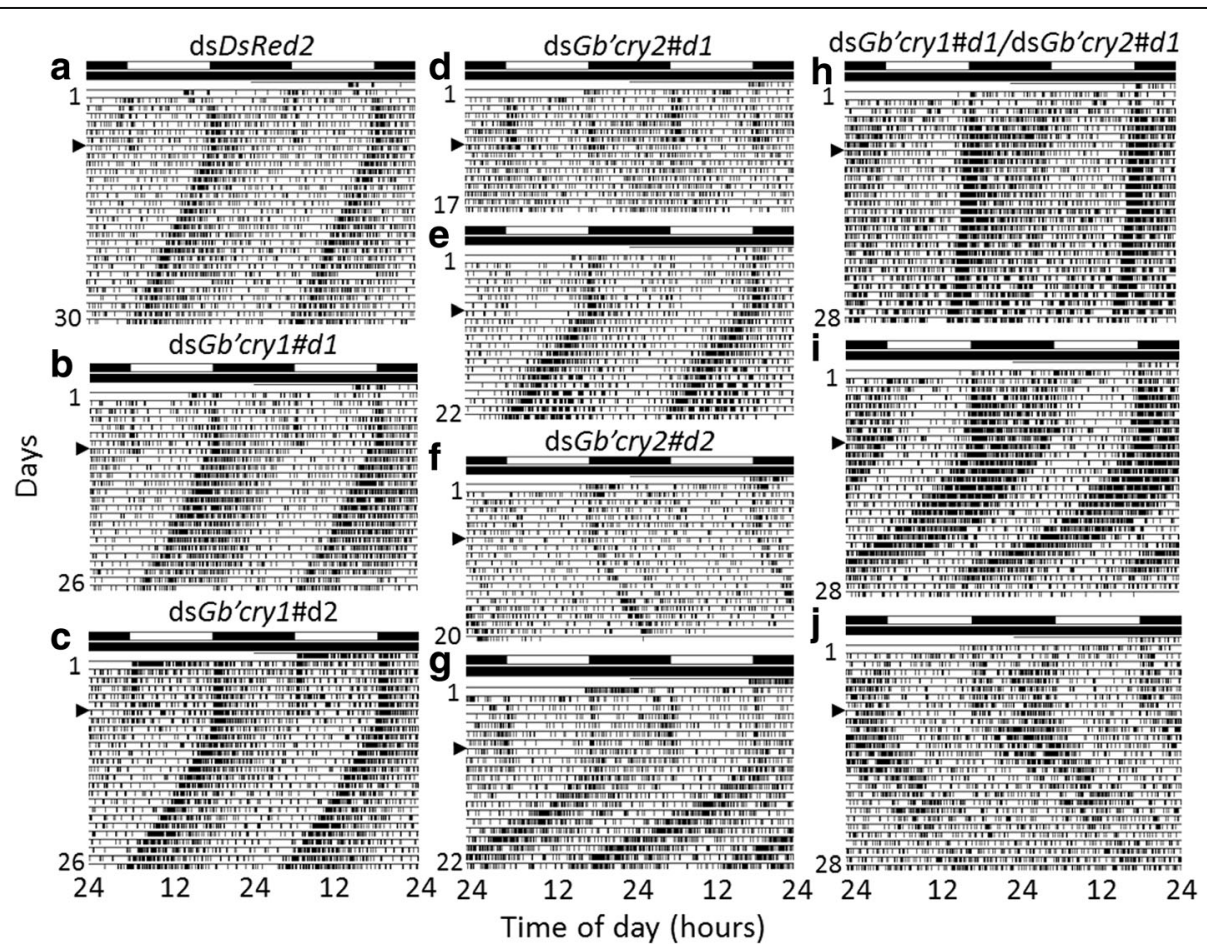

Fig. 3 Double-plotted actograms showing locomotor rhythms of crickets Gryllus bimaculatus. (a-j) Locomotor activity of DsRed2 ${ }^{\text {RAi }}$ (a), Gb'cry $7^{\text {RNAi }}$ $(\mathbf{b}, \mathbf{c}), G b^{\prime} c r y 2^{\text {RNAi }}(\mathbf{d}-\mathbf{g})$, or Gb'ry $7^{\text {RNAi }}$ and $G b^{\prime} c r y 2^{\text {RNAi }}(\mathbf{h}-\mathbf{j})$ crickets recorded under LD 12:12 and DD at a constant temperature of $25^{\circ} \mathrm{C}$. Arrowheads indicate the day when the crickets were transferred from LD to DD. Gb'cry $7^{\mathrm{RNAi}}$ crickets showed a locomotor rhythm free-running in DD with a period similar to that of DsRed2 ${ }^{\mathrm{RNAi}}$ crickets $(\mathbf{a}-\mathbf{c})$, but Gb'ry $2^{\mathrm{RNAi}}$ and Gb'cry $1 / G b^{\prime} c r y 2$ double RNAi crickets showed variable free-running periods (d-j). For further explanations, see the text 


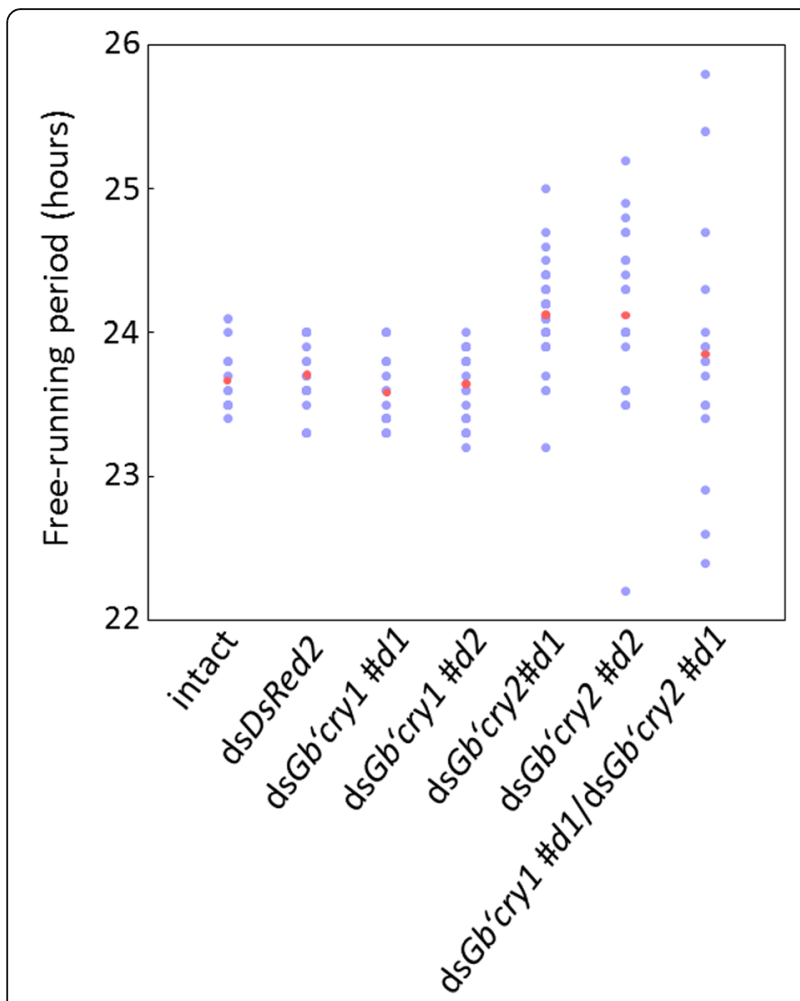

Fig. 4 Distribution of free-running periods of locomotor rhythms of individual crickets Gryllus bimaculatus. Crickets were treated with

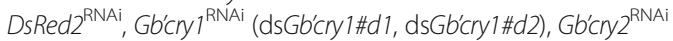
(dsGb'cry2\#d1, dsGb'cry2\#d2), or both Gb'cry $7^{\mathrm{RNAi}}$ and $G b^{\prime} c r y 2^{\mathrm{RNAi}}$ and their locomotor rhythms were measured under DD at a constant temperature of $25^{\circ} \mathrm{C}$. Blue and red dots indicate individual and average values, respectively. Gb'ry $7^{\mathrm{RNAi}}$ crickets showed a similar distribution to that of control, DsRed2 ${ }^{\mathrm{RNAi}}$ crickets. The distribution is widely ranging from 23.2 to $25.0 \mathrm{~h}$ in Gb'cry ${ }^{\mathrm{RNAi}}$ crickets, and the range is even greater in Gb'cry1/Gb'cry2 double RNAi crickets. For further explanations, see the text

ANOVA, $F_{5,23}=21.38, P<0.01$; cosinor, $P<0.05$ for Gb'tim) with a peak similar to that of untreated crickets $\left(G b^{\prime}{ }^{\prime} r y 2\right)$ or advanced by about $4-8$ h to peak during late day to early night (Gb'tim); Gb'per also showed a similar rhythmic pattern to Gb'tim, showing a significant fluctuation (ANOVA, $F_{5,21}=3.59, P<0.05$ ), but no daily rhythm was detected by the single cosinor method $(P>$ 0.05) (Fig. 5a). The results of Gb'ry2 RNAi are shown in Fig. 5b. No clear effect was observed on Gb'cry1 mRNA levels. Gb'tim showed a rhythm (ANOVA, $F_{5,20}=3.90, P<$ 0.05 ; cosinor, $P<0.05$ ) with a peak at late day (ZT10), while Gb'per fluctuated around a medium range of the control, with no significant daily rhythm (ANOVA, $F_{5,21}=$ 1.54, $P>0.23$; cosinor, $P>0.05)$.

When Gb'cry1 and Gb'cry 2 were simultaneously knocked down, both Gb'ry1 and Gb'rry2 showed no significant daily rhythm (Fig. 5c: ANOVA, $\mathrm{F}_{5,22}=0.54, P>0.73$; cosinor, $P>0.05$ for Gb'cry 1 and ANOVA, $F_{5,22}=2.14 P>$ 0.11 ; cosinor, $P>0.05$ for Gb'cry2). Gb'per showed an arrhythmic expression pattern with mRNA levels around a medium range of control (ANOVA, $\mathrm{F}_{5,23}=2.02, P>0.12$; cosinor, $P>0.05$ ), while Gb'tim maintained a robust oscillation with a peak slightly advanced to peak at ZT14 (Fig. 5c: ANOVA, $F_{5,20}=9.36, P<0.01$; cosinor, $P<0.05$ ).

\section{Gb'cry1 and Gb'cry2 regulates CLK/CYC transcriptional activity}

We examined the role of $G b^{\prime} c r y 1$ and G'cry2 in the molecular clockwork using cell-based assay. Various combinations of Gb'ry1 and Gb'cry $2 \mathrm{a} \sim \mathrm{f}$ were transfected into S2 cells together with Drosophila Clk and Drosophila tim-Luc. When Gb'ry2c was cotransfected with Gb'cry1 or Gb'cry2f, transcriptional activity of CLK/CYC was significantly suppressed (Dunnett's test, $P<0.05$; Fig. 6). CLK/CYC transcriptional activity was enhanced when Gb'rry2e was co-transfected with Gb'rry2a or Gb'rry2d (Dunnett's test, $P<0.05$; Fig. 6). Transfection in other combination had no significant effects on transcriptional activity through the E-box.

\section{Effects of RNAi of Gb'per, Gb'tim, Gb'Clk and $G b^{\prime} c y c$ on $G b^{\prime} c r y 1$ and $G b^{\prime} c r y 2$ expression}

To investigate the relationship of Gb'cry 1 and Gb'cry 2 with a Gb'per/Gb'tim loop, we examined the effects of RNAi of Gb'per, Gb'tim, Gb'Clk and Gb'cyc on the expression levels of Gb'ry1 and G'cry2. These treatments have shown that molecular oscillation of respective gene is eliminated [9-12]. The RNAi of these clock genes had no clear effects on Gb'cry1 mRNA levels except for $G b^{\prime} c y c^{\mathrm{RNAi}}$ at ZT22, at which a significant increase in Gb'cry1 mRNA was observed (Fig. 7a). The same treatments, however, significantly affected Gb'cry2 mRNA expression (Fig. 7b). $G b^{\prime}$ per ${ }^{\mathrm{RNAi}}$ upregulated the Gb'ry2 mRNA levels to raise the bottom level to exceed the peak level of control crickets and lead to arrhythmic expression (ANOVA, $\mathrm{F}_{5,18}=1.12, \quad P>0.39 ;$ cosinor, $\left.P>0.05\right)$. In $G b^{\prime} C l k^{\mathrm{RNAi}}$ crickets, Gb'cry2 mRNA showed no expression rhythm, remaining at the median range (ANOVA, $F_{5,17}=2.01, P>$ 0.14 ; cosinor, $P>0.05)$. G' ${ }^{\prime}$ cry 2 mRNA retained a daily rhythm with a normal level in Gb'tim ${ }^{\text {RNAi }}$ and Gb'cyc ${ }^{\mathrm{RNAi}}$ crickets (ANOVA, $F_{5,23}=4.62, P<0.01$; cosinor, $P<0.05$ for Gb'tim ${ }^{\text {RNAi }}$ and ANOVA, $F_{5,17}=7.02, P<0.01$; cosinor $P<0.05$ for $G b^{\prime} c y c^{\mathrm{RNAi}}$ ), but the amplitude was slightly attenuated with the increased levels during daytime. These results strongly suggest that rhythmic expression of Gb'ry2 is regulated by a mechanism other than the Gb'per/Gb'tim loop.

\section{Discussion}

\section{$G b^{\prime} c r y 1$ and $G b^{\prime}$ cry 2 genes of the cricket}

In insects, two cry genes have been identified: one is the Drosophila-type cry 1 and the other is the mammaliantype cry2 [3, 22]. The present study showed that the 

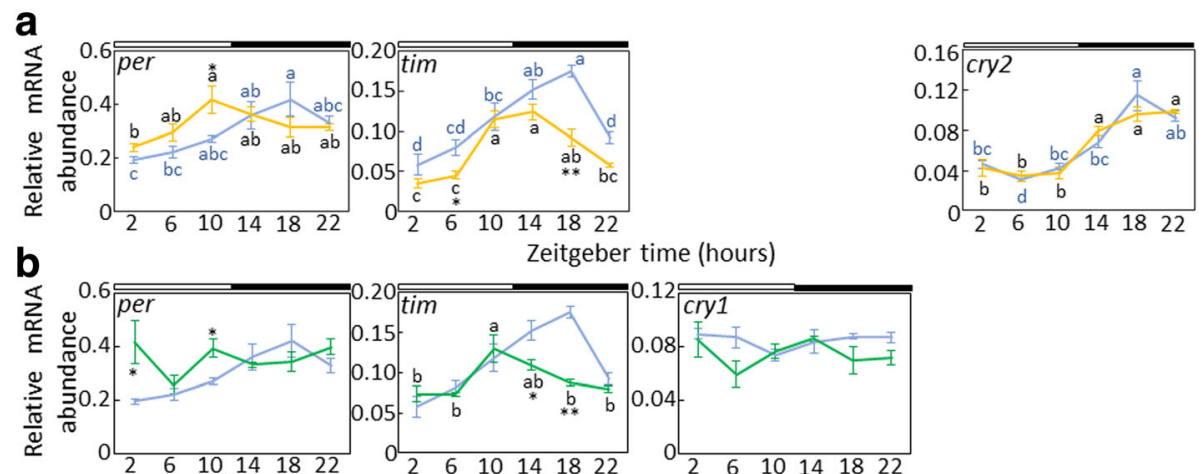

Zeitgeber time (hours)
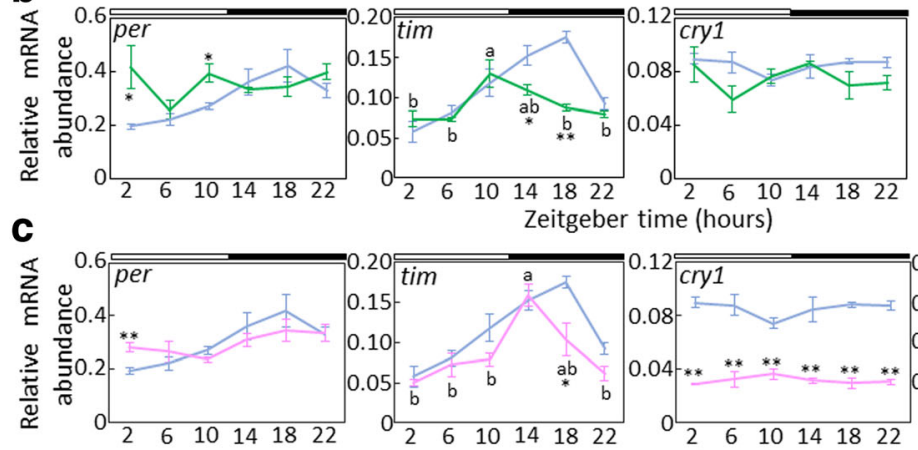
Zeitgeber time (hours)
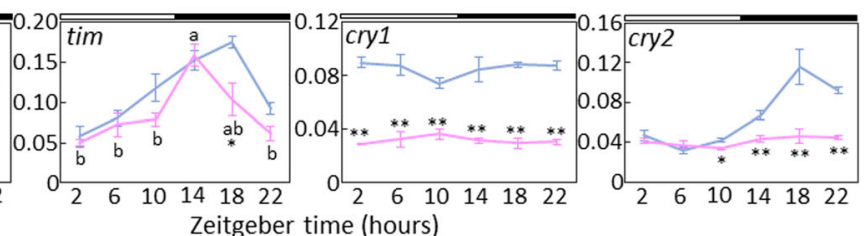

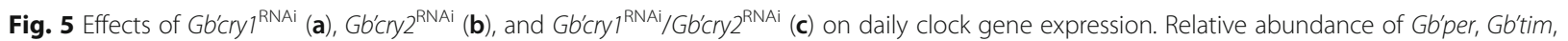

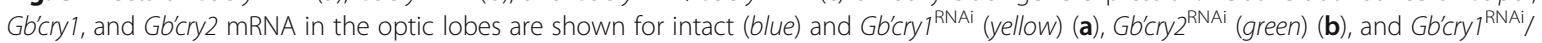
$\mathrm{Gb}^{\prime} c r y 2^{\text {RNAi }}$ (pink) adult male crickets (Gryllus bimaculatus) (c). The data for intact Gb'cry1 and Gb'cry2 mRNA are replotted from Fig. 2. In dsRNAinjected crickets, the optic lobes were collected about seven days after the dsRNA injection. The abundance of mRNA was measured by quantitative real-time RT-PCR with total RNA extracted from the optic lobes. The data collected from 3 and 5 independent experiments were averaged and plotted as mean \pm SEM for dsRNA-injected and intact crickets, respectively. The abundance of Gb'rp/18a mRNA was used as an internal reference. Asterisks indicate significant difference between intact and treated crickets $\left({ }^{*} P<0.05,{ }^{* *} P<0.01, t\right.$-test). Different letters indicate that values are significantly different with each other (ANOVA followed by Tukey-test, $P<0.05$ ). For further explanations, see the text

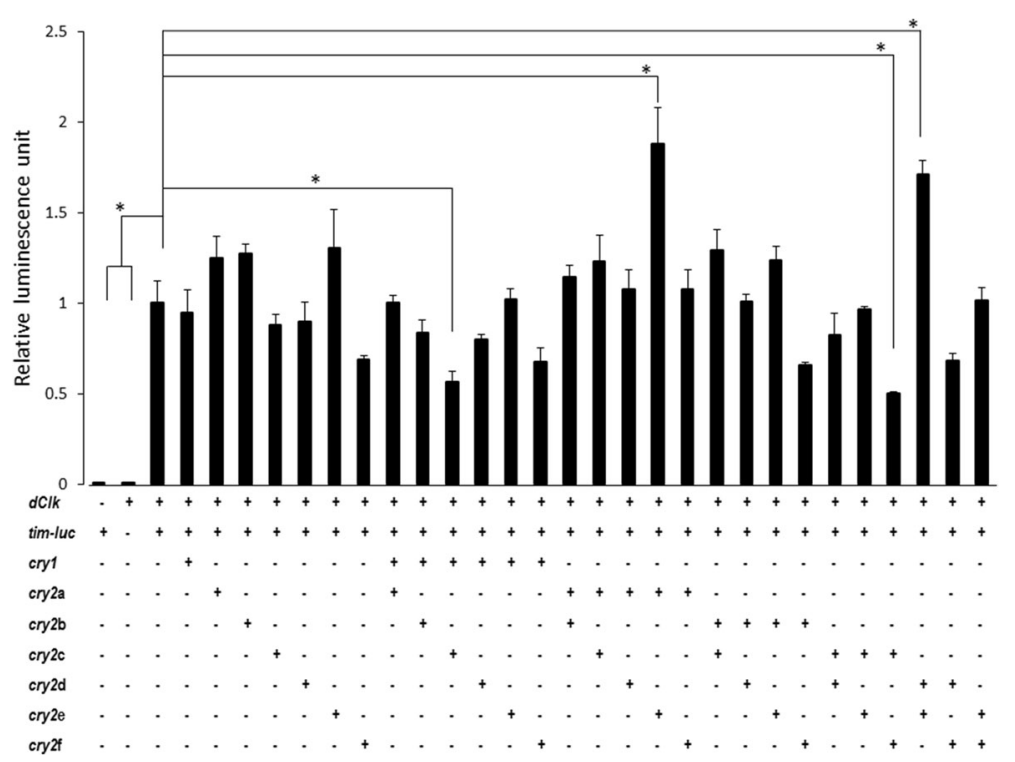

Fig. 6 Suppressive effects of $G b^{\prime} C R Y 1$ or $G b^{\prime} C R Y 2$ proteins on tim transcription. Relative luciferase activity of tim-luc was measured in the presence of $100 \mathrm{ng}$ of pAc-Clk with various combinations of the indicated pAc5.1B-Gb'cryl, pAc5.1B-Gb'cry2a, - b, -c, - d, -e, or -f expression plasmids. Signals were normalized to Rluc activity and then to the control signal of cells transfected with $100 \mathrm{ng}$ of tim-luc and pAc-Clk. Error bars represent SEM $(n=3)$. A combination of Gb'cry2b and Gb'cry2c suppressed the transcription of E-box genes. Asterisks represents $P<0.01$ versus control (Dunnett's multiple range test) 


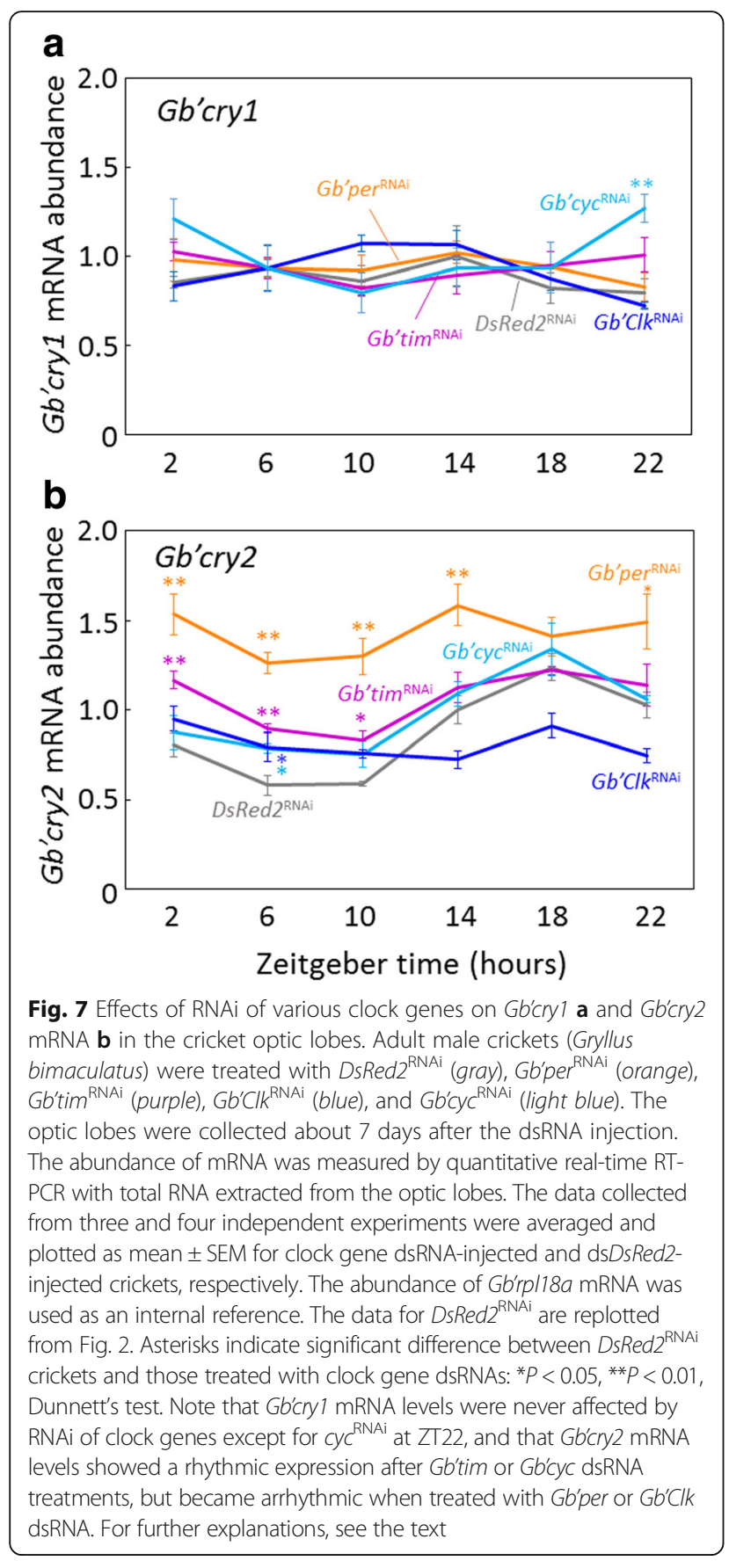

cricket, Gryllus bimaculatus also has two cry genes. Gb'cry2 has six splicing variants with variations in exons 2, 4, 6 and 10. The expression levels were different among those variants and greatest in Gb'rry2f with an exon 4, long exons 2 and 6, and a short exon 10. Their daily expression profiles in the optic lobe are similar to those in other insects; Gb'cry2 showed a rhythmic expression with a peak around mid-night, while Gb'cry1 is rather constitutively expressed throughout a day with a slight reduction at the late day phase. The reduction of
Gb'ry1 may be caused by a light-induced suppression mechanism that is known to regulate cry1 in other insects [3]. Gb'cry1 expression is most likely independent of the circadian clock, since RNAi of clock genes had nearly no effect on Gb'cry1 mRNA levels (Fig. 7a).

\section{Role of Gb'cry1 and Gb'cry2 in the cricket's clock}

In flies and butterflies, CRY1 is supposed to be a blue light receptor, and resets the clock by leading to a degradation of TIMELESS protein in a light-dependent manner [4, 6, 23]. Our results, however, clearly show that $G b^{\prime}{ }^{\prime} r y 1^{\text {RNAi }}$ crickets synchronized to the given light dark cycle as robustly as the control crickets, suggesting that Gb'CRY1 is not the major photoreceptor for entrainment of the clock. This finding is consistent with our previous reports that the compound eye is the only photoreceptor necessary for light entrainment of the cricket clock [24] and that opsin-long wavelength (opsin$L W$ ) expressed in the compound eye plays a major role in photic entrainment [25]. Similarly Gb'cry2 is apparently not a major photoreceptor for entrainment, because $G b^{\prime}{ }^{\prime} r y 2^{\mathrm{RNAi}}$ crickets also robustly synchronized to light cycles, similar to the case for Gb'cry $1^{\mathrm{RNAi}}$ crickets. However, we could not exclude the possibility that the two cry genes play subtle roles in photic entrainment. To draw a conclusion on the involvement of these genes in photic entrainment, careful examination of entrainability in Gb'cry $1^{\mathrm{RNAi}}$ and Gb'cry $2^{\mathrm{RNAi}}$ crickets is needed.

On the free-running rhythm in DD, Gb'cry $2^{\mathrm{RNAi}}$ had significant effects; the treated crickets showed variable free-running periods, suggesting that Gb'cry2 plays a significant role in the clock oscillatory mechanism. In contrast, Gb'ry $1^{\mathrm{RNAi}}$ had no clear effect, and the treated crickets showed a free-running rhythm with a period similar to that of control crickets treated with dsDsRed2. However, Gb'ry $1^{\text {RNAi }}$ enhanced the variability of the free-running period of G $b^{\prime} c r y 2^{\mathrm{RNAi}}$ crickets (Figs. 3, 4), suggesting that Gb'ry1 and Gb'cry2 cooperate to determine the free-running period.

\section{$G b^{\prime} C R Y s$ are transcriptional repressors}

The present study showed that Gb'CRYs play in the cricket molecular clockwork as transcriptional repressors (Fig. 6). We have shown for the first time that Gb'cry2 has six splicing variants and that none has a repressor activity alone. The repressor activity was evident only when G'cry2c was co-expressed with Gb'cry1 or Gb'cry $2 \mathrm{f}$ (Fig. 6). The requirement of two types of CRYs contrasts to mammalian and other insect clocks where a single molecular species of CRY can work as transcriptional repressor [3, 26, 27]. Since Gb'cry2c, Gb'cry2f, and Gb'cry1 commonly have ICAT and RD-2b, the former is likely important for repression of CLK/CYC transcriptional activity [28] and the latter may be required for the 
nuclear translocation [29]. Gb'cryl's role as a transcriptional repressor in combination with Gb'cry2 (Fig. 6) is reminiscent of a finding from peripheral tissues of Drosophila, in which cry1 is also known to function as a transcriptional repressor [23, 30]. For example, overexpression of cry 1 and per in the Drosophila compound eye represses CLK/CYC-activated transcription [30]. However, the Gb'cryl's role in transcriptional regulation is contrast to an earlier report on Anopheles gambiae, Antheraea pernyi, and Danaus plexippus, in which cry 1 has no repressor activity [3]. The role of $c r y 1$ in the core clock mechanism may be lost in these insects, in which $c r y 2$ plays a major role as a transcriptional repressor. However, we also suggest that the role of $c r y 1$ should be carefully reexamined in those insects, since Yuan et al. [3] did not examine the role of cry1 in combination with cry2.

The role of Gb'CRYs as a component in the clock machinery partly explains the changes of free-running periods in $G b^{\prime} c r y 2^{\mathrm{RNAi}}$ crickets. Even after RNAi treatment it is expected that a small amount of mRNA will be expressed. The variable amount of expressed Gb'cry2 probably resulted in variable free-running periods among individuals treated by $G b^{\prime} c r y 2^{\mathrm{RNAi}}$ or G $b^{\prime} c r y 1 /$ Gb'cry2 double RNAi (Fig. 4) as suggested for CRYs in the mammalian suprachiasmatic nucleus $(\mathrm{SCN})$ clock [31]. In $G b^{\prime}{ }^{\prime} r y 1^{\mathrm{RNAi}}$ crickets, the lack of Gb'cry1 may be compensated by another complex formed by $G b^{\prime} \mathrm{CRY} 2 \mathrm{c}$ and $G b^{\prime}$ CRY2f, which would account for the lack of any observable significant changes in this period.

Our reporter assay also showed that CLK/CYC transcriptional activity was enhanced when G'cry2e was co-expressed with G'cry2a or G'cry2d. Although this possibility cannot be ruled out, their role as transcriptional activator may be negligible, since Gb'cry2e was expressed at only trace levels and the expression of Gb'cry2a and Gb'cry2d was also quite low (Fig. 2c).

Another possible role of G'cry 2 may be as a coupling factor between clock neurons. This hypothesis is based on the observation that $G b^{\prime} c r y 2^{\mathrm{RNAi}}$ crickets sometimes show a rhythm dissociation into some components running with different free-running periods in DD (Fig. 3d). The change in coupling strength by $G b^{\prime} c r y 2^{\mathrm{RNAi}}$ may affect in variable degrees the free-running period of the locomotor rhythm. This may represent a parallel case with that described in reports on mammalian clocks in the SCN [32, 33]. Some of the Gb'CRY2 variants might play a role in this coupling and/or period change in individual clock neurons caused by $G b^{\prime} c r y 2^{\mathrm{RNAi}}$ may indirectly affect the coupling. This issue remains to be addressed in future studies.

\section{$G b^{\prime} c r y 2$ oscillation is regulated by a mechanism different from the $G b^{\prime}$ per/Gb'tim loop}

We have shown that the cricket circadian clock includes a loop for rhythmic expression of Gb'per and
Gb'tim $[9,10]$, of which transcription is regulated by $G b^{\prime}$ CLK/Gb'CYC $[11,12]$. It has been suggested that CRY2 forms a complex with PER or the PER/TIM complex to enter the nucleus and repress the CLK/ CYC transcriptional activity in other insects $[5,6]$. However, the results of the present study suggest that Gb'CRY2 composes a loop to repress $G b^{\prime}$ CLK/Gb'CYC activity by forming complex between its variants or with $G b^{\prime} C R Y 1$, and that the loop can operate independent of the Gb'per/Gb'tim loop to some degree (Fig. 8). This hypothesis is based on the following observations. When Gb'tim was knocked down by RNAi or Gb'tim and Gb'per stopped their oscillation by $G b^{\prime} c y c^{\mathrm{RNAi}}$ [12], Gb'cry2 maintains its oscillation within a normal range but with a slightly attenuated amplitude (Fig. 7). Alternatively when Gb'ry2 is knocked down, Gb'tim maintained its oscillation. Our data suggest that the circadian locomotor rhythm is expressed when either Gb'cry2 or Gb'tim is rhythmically expressed.

However, there must be an inter-connection between the assumed Gb'ry2 loop and the Gb'per/Gb'tim loop. When Gb'per or Gb'Clk was knocked down by RNAi, Gb'cry2 lost its rhythmic expression (Fig. 7), and in Gb'per $^{\mathrm{RNAi}}$ crickets the mRNA levels of G'cry2 were significantly increased, with the trough level nearly equivalent to the peak level of DsRed $2^{\mathrm{RNAi}}$ crickets (Fig. 7). Alternatively when Gb'cry2 was knocked down, Gb'per lost the rhythm but Gb'tim showed a phase

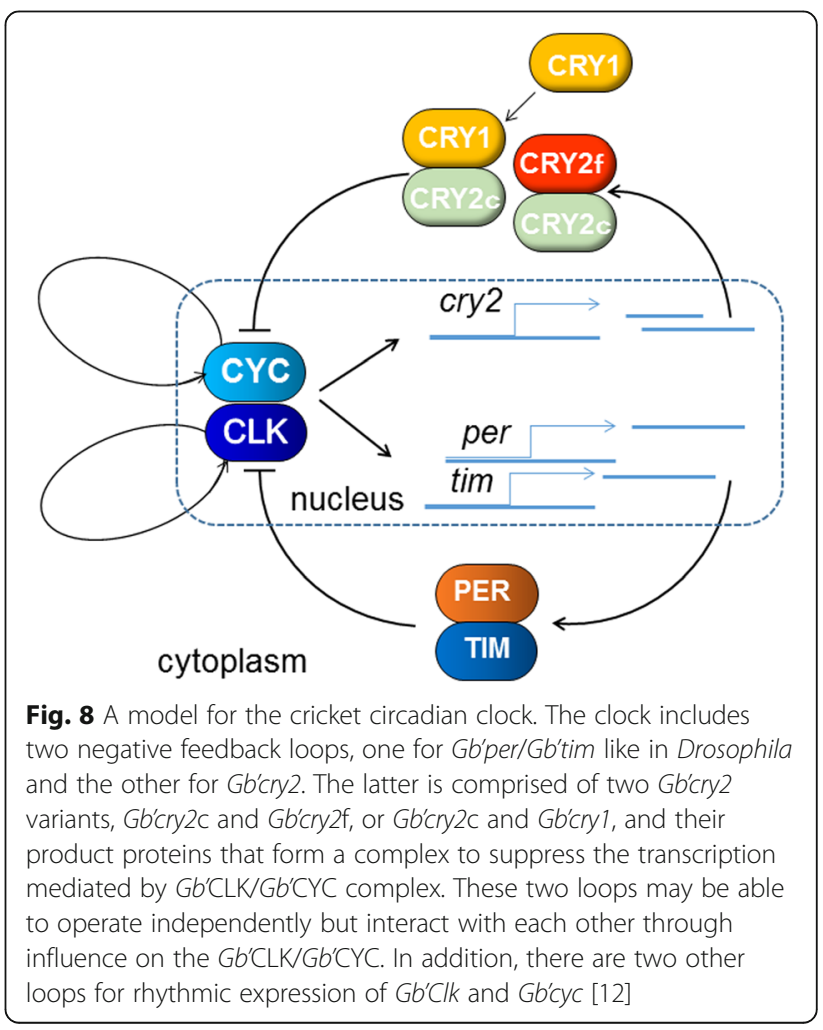


advance of its expression rhythm (Fig. 5). Although further detailed study is necessary to understand the underlying mechanism, these data suggest that some coupling mechanism exists between the two loops. For Gb'per, Gb'tim and Gb'cry2, Gb'CLK/Gb'CYC may be a common transcriptional activator. Thus, the two loops may couple with $G b^{\prime} \mathrm{CLK} / G b^{\prime} \mathrm{CYC}$ as a hinge (Fig. 8). The transcriptional activity of $G b^{\prime} \mathrm{CLK} / G b^{\prime} \mathrm{CYC}$ may be suppressed by both Gb'PER/Gb'TIM complex and $G b^{\prime} \mathrm{CRY} 1 / G b^{\prime} \mathrm{CRY} 2$ or $G b^{\prime} \mathrm{CRY} 2$ complexes. Knock-down of either Gb'cry1 or Gb'cry2 weakens the repression of CLK/CYC, resulting in the upregulation of Gbper mRNA. The transcription of Gb'tim and Gb'cry 2 may require stronger transactivation than Gb'per, and thus Gb'tim and Gb'cry2 mRNA maintained rhythmic expression even after knockdown of Gb'cry1/Gb'cry2 and Gb'tim, respectively.

\section{Conclusion}

The cricket clock consists of at least four loops, each of which regulates rhythmic expression of Gb'per and Gb'tim, Gb'Clk, Gb'cyc, and Gb'cry2 [9, 10, 12] (Fig. 8). The clock system appears to be more complex than those of Drosophila and other insects, which basically include three loops for rhythmic expression of per and tim, Clk or cyc, and clockwork orange [1,34]. The cricket is a hemimetabolous insect, and therefore more primitive than holometabolous insects, including Diptera (Drosophila), Lepidoptera (Danaus, Antheraea), and Hymenoptera (Apis). It may thus possess a more primitive clock machinery, of which some components may have changed or been lost in the course of phylogenetic development in higher insects.

The cricket's clock possesses some properties common to mammalian clock. Gb'cyc shows a rhythmic expression similar to that of its mammalian homologue, Bmal1 [12]. Both Gb'rry1 and Gb'cry2 are involved in the clock machinery. The reset mechanism is exclusively relies on retinal photoreceptors $[24,25,35]$. Similarly the firebrat Thermobia domestica, one of the most primitive ametabolous insects, has a mammalian-like clock machinery in which $c y c$ is rhythmically expressed [36, 37]. These findings suggest that the ancestral insect clock resembled the mammalian clock and subsequently diversified into various types [1].

The evolution and diversification of insect cry genes remains a challenging issue. There is a variation for presence and absence of cry genes. Although many insects have both $c r y 1$ and $c r y 2$, some dipteran species lack $\operatorname{cry} 2$ and some coleopteran and hymenopteran species lack cry1 $[1,38]$. The present study revealed that $G b^{\prime} \mathrm{CRY} 1$ is not a photoreceptor, but rather a component of the clock and that Gb'cry2 has splicing variants, providing another example of diversification of cryl and cry2. These findings make it difficult to resolve the evolution or diversification of insect cry genes. To deal with this issue, extensive comparative studies are desired across phylogenetically diverse species, including hemimetabola and ametabola.

\section{Abbreviations \\ ANOVA: Analysis of variance; Clk: Clock; CLK: CLOCK; cry: cryptochrome; CRY: CRYPTOCHROME; cry1: cryptochrome 1; CRY1: CRYPTOCHROME 1; cry2: cryptochrome 2; CRY2: CRYPTOCHROME 2; cyc: cycle; CYC: CYCLE; DD: Constant darkness; dsRNA: Double stranded RNA; Gb: Gryllus bimaculatus; ICAT: Inhibition of CLOCK-ARNTL-mediated transcription domain; LD: Light/ dark; opsin-LW: Opsin-long wavelength; per: period; PER: PERIOD; \\ qPCR: Quantitative real-time reverse transcription polymerase chain reaction; RACE: Rapid amplification of cDNA ends; RNAi: RNA interference; rpl18a: ribosomal protein L18a; RT-PCR: Reverse transcription polymerase chain reaction; SCN: Suprachiasmatic nucleus; tim: timeless; TIM: TIMELESS; ZT: Zeitgeber time}

\section{Acknowledgements}

The authors thank Dr. Sumihare Noji of Tokushima University for providing genomic information on the cricket.

\section{Funding}

This study was supported in part by grant-in-aid No. 15 H0440017 from JSPS.

Availability of data and materials

The datasets supporting the conclusions of this article are included within the article.

\section{Authors' contributions}

AT, TQI, SH, OU, YD and MN performed the experiments. AT and KT wrote the paper. KT, AT and YD designed the experiments. TB and TT supported molecular analysis of cry genes. All authors read and approved the final manuscript.

\section{Competing interests}

The authors declare they have no competing interests.

Ethics approval and consent to participate Not applicable.

Consent for publication

Not applicable.

\section{Publisher's Note}

Springer Nature remains neutral with regard to jurisdictional claims in published maps and institutional affiliations.

\section{Author details}

${ }^{1}$ Graduate School of Natural Science and Technology, Okayama University, 3-1-1 Tsushima-naka, Kita-ku, Okayama 700-8530, Japan. ${ }^{2}$ Graduate School of Science, Kyushu University, Fukuoka 819-0395, Japan. ${ }^{3}$ Okayama University Graduate School of Medicine, Dentistry and Pharmaceutical Sciences, Okayama 700-8558, Japan.

Received: 24 January 2017 Accepted: 30 March 2017

Published online: 06 April 2017

\footnotetext{
References

1. Tomioka K, Matsumoto A. Circadian molecular clockworks in non-model insects. Curr Opin Insect Sci. 2015;7:58-64.

2. Tataroglu O, Emery P. The molecular ticks of the Drosophila circadian clock. Curr Opin Insect Sci. 2015;7:51-7.

3. Yuan Q, Metterville D, Briscoe AD, Reppert SM. Insect cryptochromes: gene duplication and loss define diverse ways to construct insect circadian clocks. Mol Biol Evol. 2007:24:948-55

4. Ceriani MF, Darlington TK, Staknis D, Mas P, Petti AA, Weitz CJ, et al. Light-dependent sequentation of TIMELESS by CRYPTOCHROME. Science. 1999;285:553-6.
} 
5. Rubin EB, Shemesh Y, Cohen M, Elgavish S, Robertson HM, Bloch G. Molecular and phylogenetic analyses reveal mammalian-like clockwork in the honey bee (Apis mellifera) and shed new light on the molecular evolution of the circadian clock. Genome Res. 2006;16:1352-65.

6. Zhu H, Sauman I, Yuan Q, Casselman A, Emery-Le M, Emery P, et al. Cryptochromes define a novel circadian clock mechanism in monarch butterflies that may underlie sun compass navigation. PLoS Biol. 2008;6:e4.

7. Tomioka K. Chronobiology of crickets: a review. Zool Sci. 2014;31:624-32.

8. Tomioka K, Sakamoto T, Moriyama Y. RNA interference is a powerful tool for chronobiological study in the cricket. Sleep Biol Rhythms. 2009;7:144-51.

9. Moriyama Y, Sakamoto T, Karpova SG, Matsumoto A, Noji S, Tomioka K. RNA interference of the clock gene period disrupts circadian rhythms in the cricket Gryllus bimaculatus. J Biol Rhythms. 2008;23:308-18.

10. Danbara Y, Sakamoto T, Uryu O, Tomioka K. RNA interference of timeless gene does not disrupt circadian locomotor rhythms in the cricket Gryllus bimaculatus. J Insect Physiol. 2010;56:1738-45.

11. Moriyama Y, Kamae Y, Uryu O, Tomioka K. Gb'Clock is expressed in the optic lobe and required for the circadian clock in the cricket Gryllus bimaculatus. J Biol Rhythms. 2012;27(6):467-77.

12. Uryu O, Karpova SG, Tomioka K. The clock gene cycle plays an important role in the circadian clock of the cricket Gryllus bimaculatus. J Insect Physiol. 2013;59:697-704

13. Moriyama Y, Sakamoto T, Matsumoto A, Noji S, Tomioka K. Functional analysis of the circadian clock gene period by RNA interference in nymphal crickets Gryllus bimaculatus. J Insect Physiol. 2009;55:396-400.

14. Darlington TK, Wager-Smith K, Ceriani MF, Staknis D, Gekakis N, Steeves TDL, et al. Closing the circadian loop: CLOCK-induced transcription of its own inhibitors per and tim. Science. 1998;280:1599-603.

15. McDonald MJ, Rosbash M, Emery P. Wild-type circadian rhythmicity is dependent on closely spaced $\mathrm{E}$ boxes in the Drosophila timeless promotor. Mol Cell Biol. 2001;21:1207-17.

16. Ueda HR, Matsumoto A, Kawamura M, Tanimura T, Hashimoto S. Genomewide transcriptional orchestration of circadian rhythm in Drosophila. J Biol Chem. 2002;277:14048-52.

17. Matsumoto A, Ukai-Tadenuma M, Yamada RG, Houl J, Uno KD, Kasukawa T, et al. A functional genomics strategy reveals clockwork orange as a transcriptional regulator in the Drosophila circadian clock. Gene Dev. 2007; 21:1687-700.

18. Sokolove $P G$, Bushell WN. The chi square periodogram: its utility for analysis of circadian rhythm. J Theor Biol. 1978;72:131-60.

19. Schmid B, Helfrich-Förster C, Yoshii T. A new ImageJ plug-in "ActogramJ" for chronobiological analyses. J Biol Rhythms. 2011;26:464-7.

20. Kaneko M, Hall JC. Neuroanatomy of cells expressing clock genes in Drosophila: transgenic manipulation of the period and timeless genes to mark the perikarya of circadian pacemaker neurons and their projections. J Comp Neurol. 2000;422:66-94.

21. Nelson W, Tong Y, Lee J, Halberg F. Methods for cosinor-rhythmometry. Chronobiologia. 1979;6:305-23.

22. Zhu H, Yuan Q, Briscoe AD, Froy O, Casselman A, Reppert SM. The two CRYs of the butterfly. Curr Biol. 2005;15:R953-954.

23. Ivanchenko M, Stanewsky R, Giebultowicz JM. Circadian photoreception in Drosophila: functions of cryptochrome in peripheral and central clocks. J Biol Rhythms. 2001;16:205-15.

24. Tomioka K, Chiba Y. Effects of nymphal stage optic nerve severance or optic lobe removal on the circadian locomotor rhythm of the cricket, Gryllus bimaculatus. Zool Sci. 1984;1:385-94.

25. Komada S, Kamae Y, Koyanagi M, Tatewaki K, Hassaneen E, Saifullah A, et al. Green-sensitive opsin is the photoreceptor for photic entrainment of an insect circadian clock. Zool Lett. 2015;1:11.

26. Ye R, Selby CP, Chiou Y-Y, Ozkan-Dagliyan I, Gaddameedhi S, Sancar A. Dual modes of CLOCK:BMAL1 inhibition mediated by Cryptochrome and Period proteins in the mammalian circadian clock. Gene Dev. 2016;28:1989-98.

27. Langmesser S, Tallone T, Bordon A, Rusconi S, Albrecht U. Interaction of circadian clock proteins PER2 and CRY with BMAL1 and CLOCK. BMC Mol Biol. 2008;9:41

28. Ingram KK, Kutowoi A, Wurm Y, Shoemaker D, Meier R. The molecular clockwork of the fire ant Solenopsis invicta. PLoS One. 2012;7:e45715.

29. Hirayama J, Nakamura H, Ishikawa T, Kobayashi Y, Todo T. Functional and structural analyses of cryptochrome. Vertebrate CRY regions responsible for interaction with the CLOCK:BMAL1 heterodimer and its nuclear localization. J Biol Chem. 2003;278:35620-8.
30. Collins B, Massoni EO, Stanewsky R, Blau J. Drosophila CRYPTOCHROME is a circadian transcriptional repressor. Curr Biol. 2006;16:441-9.

31. Anand SN, Maywood ES, Chesham JE, Joynson G, Banks GT, Hastings MH, et al. Distinct and separable roles for endogenous CRY1 and CRY2 within the circadian molecular clockwork of the suprachiasmatic nucleus, as revealed by the FbxI3 ${ }^{\text {Afh }}$ mutation. J Neurosci. 2013:33:7145-53.

32. Ono D, Honma S, Honma K-I. Cryptochromes are critical for the development of coherent circadian rhythms in the mouse suprachiasmatic nucleus. Nat Commun. 2013:4:1666.

33. Evans JA, Pan H, Liu AC, Welsh DK. Cry $7^{-1-}$ circadian rhythmicity depends on SCN intercellular coupling. J Biol Rhythms. 2012;27:443-52.

34. Hardin PE. Essential and expendable features of the circadian timekeeping mechanism. Curr Opin Neurobiol. 2006;16:686-92.

35. Tomioka K, Okada Y, Chiba Y. Distribution of circadian photoreceptors in the compound eye of the cricket Gryllus bimaculatus. J Biol Rhythms. 1990:5:131-9.

36. Kamae Y, Tanaka F, Tomioka K. Molecular cloning and functional analysis of the clock genes, Clock and cycle, in the firebrat Thermobia domestica. J Insect Physiol. 2010;56:1291-9.

37. Kamae Y, Uryu O, Miki T, Tomioka K. The nuclear receptor genes HR3 and E75 are required for the circadian rhythm in a primitive insect. PLoS One. 2014;9:e114899

38. Zhan S, Merlin C, Boore JL, Reppert SM. The monarch butterfly genome yields insights into long-distance migration. Cell. 2011;147:1171-85.

\section{Submit your next manuscript to BioMed Central and we will help you at every step:}

- We accept pre-submission inquiries

- Our selector tool helps you to find the most relevant journal

- We provide round the clock customer support

- Convenient online submission

- Thorough peer review

- Inclusion in PubMed and all major indexing services

- Maximum visibility for your research

Submit your manuscript at www.biomedcentral.com/submit
) Biomed Central 